\title{
Selective Deposition of Lipid Membranes on Locally Anodic-Oxidized Silicon Surface
}

\author{
Masaya Nakamura, Toshinari Isono, and Toshio Ogino* \\ Graduate School of Engineering, Yokohama National University, \\ 79-5 Tokiwadai, Hodogayaku, Yokohama 240-8501, Japan
}

(Received 7 January 2011; Accepted 22 August 2011; Published 27 September 2011)

\begin{abstract}
We demonstrate control of biomolecule adsorption on $\mathrm{Si}$ oxide islands formed by local anodic oxidation using atomic force microscopy. Local anodic oxidation was performed on a thin- $\mathrm{SiO}_{2} / \mathrm{Si}$ substrate covered with an octadecyltrimethoxysilane (OTMS) film. Dipalmitoylphosphatidylcholine (DPPC) molecules were deposited on the oxide islands. Hydrophilicity of the oxide island surfaces decreased with an increase in the applied voltage. When high voltages were applied, the oxide islands laterally expanded to form round shape and more hydrophilic oxide areas formed in the periphery of the islands. Density of $\mathrm{OH}$ groups on the anodic oxide island surface, which determines the surface hydrophilicity, is changed by the applied voltage during the oxidation. Since lipid membrane formation is strongly affected by surface hydrophilicity, selective deposition of DPPC membranes was achieved. We concluded that local anodic oxidation is a useful method for controlling biomolecule adsorption through oxide surfaces with variable hydrophilicity. [DOI: 10.1380/ejssnt.2011.357]
\end{abstract}

Keywords: Atomic force microscopy; Self-assembly; Surface chemical reaction; Silicon oxides; Local anodic oxidation; Supported lipid membrane

\section{INTRODUCTION}

Control of biomolecule adsorption on a solid surface is one of the important issues in solid-based biodevices. Since biomolecule adsorption on a solid surface is caused by electrostatic and hydrophobic interactions [1], surface properties should be designed to control biomolecule adsorption on a solid surface. Among various techniques to modify surface properties, annealing is a simple method to reduce surface hydrophilicity [2]. Covering with a selfassembled monolayer (SAM) can be used to change surface functional group by selecting the head group of the SAM molecules [3]. Using photolithography [4] or electron beam lithography [5], nanopatterning of modified areas can be achieved. These methods are used to modify a solid surface for protein [6] or nucleic acid [7] patterning. However, those methods can be used only to form chemically discrete patterns that are determined by the molecule species. To control biomolecule adsorption, it is desirable that surface chemistry is continuously regulated corresponding to molecule species.

Surface modification using local anodic oxidation by atomic force microscopy (AFM) was first reported by Thundat et al. [8]. In this technique, the surface layer is locally oxidized by applying a negative voltage to the conductive AFM tip with respect to the sample surface $[8,9]$. Nano- and microscaled oxide patterns can be grown on various metal or semiconductor substrates [10-16]. Oxide thickness and lateral size can be changed by the oxidation conditions [17-19]. Local anodic oxidation is known as a technique not only to fabricate nanostructures or patterns on the substrate, but also to control hydrophilicity on the oxide island surfaces by selecting the applied voltages during the oxidation $[20,21]$. Surfaces oxidized by voltages as low as $7 \mathrm{~V}$ exhibit a frictional force larger than that oxidized by voltages higher than $8 \mathrm{~V}$, where the frictional force was measured by AFM as a magnitude of cantilever torsion during the scan [22]. Frictional force measured by

*Corresponding author: togino@ynu.ac.jp
AFM in air is generally determined by the meniscus force between the surface and the hydrophilic cantilever [2326]. Water molecules of the meniscus attract each other and prevent cantilever motion. Therefore, larger frictional force is obtained on a hydrophilic surface compared with a hydrophobic surface. Since surface hydrophilicity of the oxides is usually related to the density of $\mathrm{OH}$ groups on the surface $[2,27]$, we can conclude that the density of $\mathrm{OH}$ groups on the anodic oxide can be controlled by the applied voltage during local anodic oxidation [20,21]. The chemical states of the anodic-oxidized surface are basically kept the same in aqueous environment as in air [21]. These are important advantages of the local anodic oxidation when applying it to surface modification for control of biointerfaces because surface chemistry influences biomolecule adsorption $[2,3]$.

In this report, we show experimental results of selective deposition of lipid membranes on oxide islands formed by local anodic oxidation. The oxide islands were formed by different applied voltages to change the surface chemistry. Neutral lipid molecules were used to eliminate electrostatic interaction. The base surface was covered with a $\mathrm{CH}_{3}$-terminated SAM film to avoid nonselective formation of a lipid bilayer. We discuss how hydrophilic/hydrophobic interaction between the oxide island and the lipid molecules affects lipid membrane formation. We confirm that local anodic oxidation is a suitable method to control local surface chemistry toward bio or medical applications.

\section{EXPERIMENTAL PROCEDURES}

Silicon wafers were cleaned with a mixture of $\mathrm{H}_{2} \mathrm{SO}_{4}$ and $\mathrm{H}_{2} \mathrm{O}_{2}$, and chemical oxide layers were formed during this process. Octadecyltrimethoxysilane (OTMS) films were deposited on the $\mathrm{SiO}_{2} / \mathrm{Si}$ surfaces by the vapor-phase deposition method to make the surface hydrophobic by the alkyl chains. We refer to this SAM-terminated sample as OTMS $/ \mathrm{SiO}_{2} / \mathrm{Si}$ surface. Local anodic oxidation was performed using the contact mode of AFM (SPI4000/ESweep; Seiko Instruments Inc.) equipped with a $1.6 \mathrm{~N} / \mathrm{m}$ rhodium-coated Si cantilever in air of $30 \%$ ambient hu- 


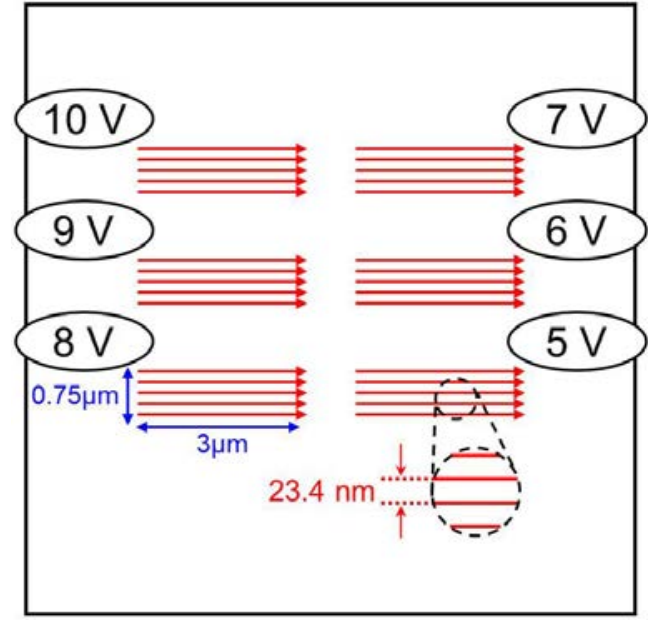

FIG. 1: Schematic of the experimental procedure of local anodic oxidation. The values in the circles indicate the applied voltages between the tip and the surface during anodic oxidation.

midity at room temperature. The apex diameter of the AFM tip was approximately $60 \mathrm{~nm}$. The applied voltage for oxidation was regulated from 5 to $10 \mathrm{~V}$, and the scanning speed was $1200 \mathrm{~nm} / \mathrm{s}$. Rectangular oxide islands were obtained by repeating line-scan oxidation accompanied with a tip shift by $23.4 \mathrm{~nm}$, as shown in Fig. 1. Under this scan condition, oxidized areas formed by a single line scan overlap with the adjacent areas due to expansion of the oxidized areas.

After the anodic oxidation, we observed surface topography of the substrate by the tapping mode of AFM without replacement of the cantilever. We then took frictional force images of the surfaces using the contact mode of AFM with a $0.08 \mathrm{~N} / \mathrm{m} \mathrm{Si}_{3} \mathrm{~N}_{4}$ cantilever under almost the same conditions as the local anodic oxidation. A frictional force image is taken by detecting the torsion of the cantilever during the scan [22]. Contrast in each frictional force image is relative with respect to the frictional force on the base OTMS surface.

Dipalmitoylphosphatidylcholine (DPPC) molecules, purchased from Avanti Polar Lipids Inc., were dissolved in chloroform, and the solvent was removed by a vacuum-drying to obtain lipid thin films. A buffer solution $\left(150 \mathrm{mM} \mathrm{KCl}, 1.0 \mathrm{mM} \mathrm{CaCl}_{2}\right.$, $10 \mathrm{mM}$ N-2-hydroxyethylpiperazine-N'-2-ethanesulfonic acid (HEPES)/KOH, pH 7.4) was added to the lipid films and warmed up above the gel-liquid crystal transition temperature $\left(41^{\circ} \mathrm{C}\right.$ for DPPC). HEPES was purchased from EMD Biosciences, Inc. and the other reagents were from Kanto Chemical Co., Inc. All reagents were used without further purification. Vesicles were assembled by stirring the lipid solution and then small unilamellar vesicles were prepared by mild sonication using a bath-type sonicator. The size of vesicles is estimated to be about 10-50 $\mathrm{nm}$ from the reported experimental results using similar conditions [28]. To accelerate the vesicle rupture on the substrate surface, the lipid solution temperature was kept at $60^{\circ} \mathrm{C}$ during the incubation for $1 \mathrm{~h}$.

After the lipid membrane deposition, we observed surface topography of the substrate using the tapping mode
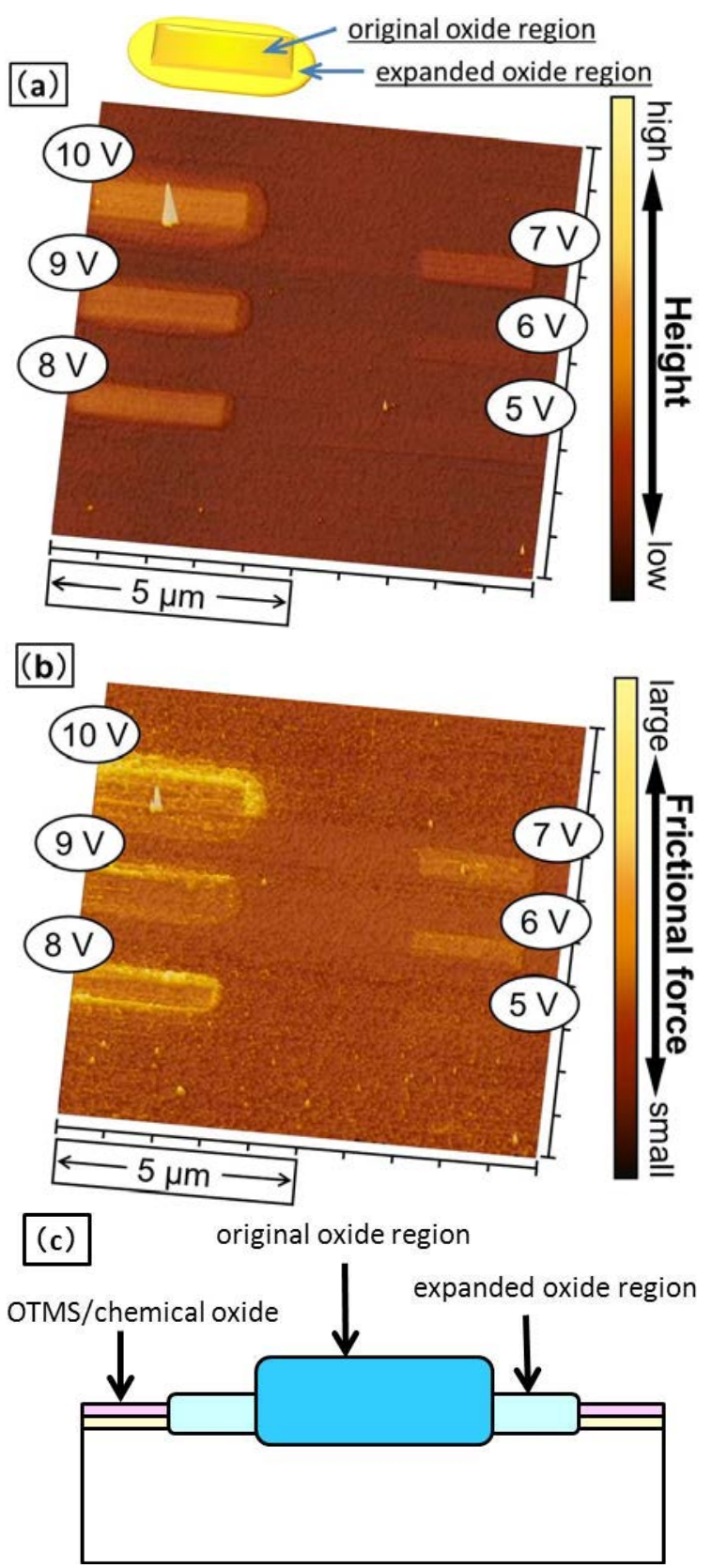

FIG. 2: AFM images of locally anodic-oxidized islands on the OTMS $/ \mathrm{SiO}_{2} / \mathrm{Si}$ surface: (a) topography and (b) frictional force image. The values indicate the applied voltages between the tip and the surface during anodic oxidation. (c) Crosssectional sketch of a formed oxide island.

of AFM with a $1.6 \mathrm{~N} / \mathrm{m}$ Si cantilever, whose apex diameter was approximately $40 \mathrm{~nm}$, in the same buffer solution. To confirm the presence of the lipid membranes, the oxide island surface was scratched by the AFM tip.

\section{RESULTS}

Figure 2 shows AFM images of (a) surface topography and (b) frictional force image in air after the local anodic oxidation on an OTMS/SiO $/$ Si surface. The values shown in Fig. 2 are the applied voltages in the oxidation. When relatively high voltages ( 8 to $10 \mathrm{~V}$ ) were 
TABLE I: Height of oxide islands formed at various applied voltages.

\begin{tabular}{ccc}
\hline \hline $\begin{array}{c}\text { Applied voltage } \\
{[\mathrm{V}]}\end{array}$ & \multicolumn{2}{c}{ Oxide height [nm] } \\
Original oxide & $\begin{array}{c}\text { Extended oxide } \\
\text { region }\end{array}$ & region \\
\hline 10 & 2.1 & 1.0 \\
9 & 1.0 & 0.8 \\
8 & 0.9 & 0.5 \\
7 & 0.6 & - \\
6 & 0.5 & - \\
5 & - & - \\
\hline \hline
\end{tabular}

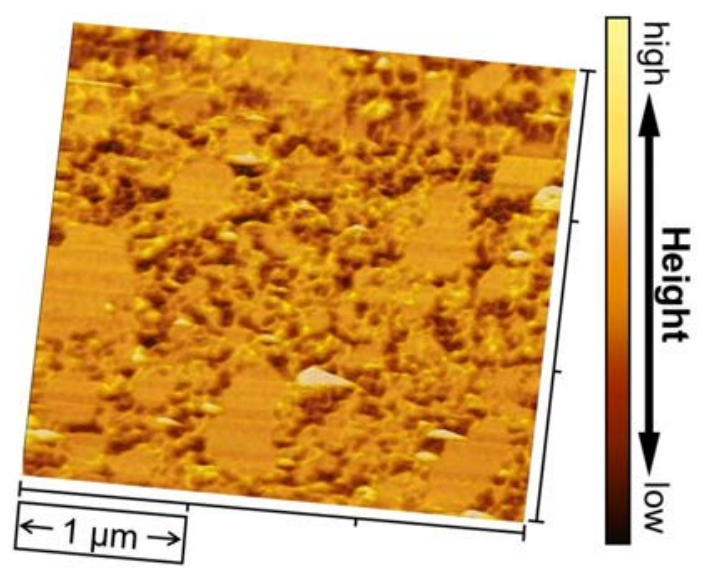

FIG. 3: AFM images of chemically oxidized $\mathrm{SiO}_{2} / \mathrm{Si}$ surface after the DPPC deposition in a buffer solution.

applied, oxide areas were expanded, and their shape became round. To distinguish the oxide areas, we refer to the rectangular thick oxide island grown just below the tip as original oxide region and round oxide area outside the original oxide region as expanded oxide region, as shown in Fig. 2(c). Height of the oxide islands and size of the expanded oxide regions increased with an increase in the applied voltage, as shown in Table I. In Fig. 2(b), the darker regions correspond to smaller frictional force, and therefore, to less hydrophilic areas. When the applied voltage was $5 \mathrm{~V}$, the surface was not oxidized. The frictional force on the oxide islands formed at voltages above $6 \mathrm{~V}$ is larger than that on the base surface covered with a hydrophobic SAM film. Frictional force on the original oxide regions decreases with an increase in the applied voltage. On the other hand, the frictional force on the expanded oxide region was much larger than that on the original oxide region. It indicates that the expanded oxide region is more hydrophilic than the original oxide region though both areas were simultaneously formed.

Figure 3 shows an AFM image of DPPC bilayers on a chemically oxidized $\mathrm{Si}$ surface as the control experiment. Theoretically expected height of DPPC bilayer, about $5 \mathrm{~nm}$, was obtained. Figure 4(a) shows an AFM image of a locally oxidized OTMS $/ \mathrm{SiO}_{2} / \mathrm{Si}$ surface taken in a buffer solution after lipid membrane deposition. Figures 4(b)-(f) are magnified AFM images around the individual oxide islands formed at (b) $10 \mathrm{~V}$, (c) $9 \mathrm{~V}$, (d) $8 \mathrm{~V}$, (e) $7 \mathrm{~V}$, and (f) $6 \mathrm{~V}$, respectively. Figure 5 shows cross-
TABLE II: Lipid membrane formation on oxide islands formed at various applied voltages.

\begin{tabular}{ccc}
\hline \hline $\begin{array}{c}\text { Applied voltage } \\
{[\mathrm{V}]}\end{array}$ & $\begin{array}{c}\text { Membrane formation } \\
\text { Original oxide } \\
\text { region }\end{array}$ & $\begin{array}{c}\text { Extended oxide } \\
\text { region }\end{array}$ \\
\hline 10 & $\times$ & $\bigcirc$ \\
9 & $\bigcirc$ & $\bigcirc$ \\
8 & $\bigcirc$ & $\bigcirc$ \\
7 & $\bigcirc$ & - \\
6 & $\times$ & - \\
5 & - & - \\
\hline \hline
\end{tabular}

sectional views on an oxide island formed at $9 \mathrm{~V}$ before and after the DPPC deposition. Membrane structures were formed on both the original oxide region and the expanded oxide region. Observed height of the membrane was about $2 \mathrm{~nm}$. On the other hand, no membrane structure was found on the base SAM surface. Spikes in the cross-section are thought to be lipid precipitates. From height measurement on the oxide islands before and after the lipid deposition, it was found that the membrane formation depends on the region, original or the expanded oxide regions, as shown in Table II. For example, lipid membrane is observed on the expanded oxide region but not on the original oxide region when the applied voltage was $10 \mathrm{~V}$. This is confirmed by the result of scratch on the oxide island formed at $10 \mathrm{~V}$, as shown in Fig. 6. The rectangular area enclosed by the broken white line indicates the scratched area. The membrane structure on the expanded oxide region was removed by the scratch, but the surface on the original oxide region was unchanged. On the original oxide islands formed at voltages between 7 and $9 \mathrm{~V}$, lipid membranes formed. Thus, site-selective deposition of DPPC membranes on the anodic-oxide islands was confirmed.

\section{DISCUSSION}

A lipid bilayer generally forms on a hydrophilic surface because the surface of lipid bilayer is hydrophilic. However, lipid bilayer formation by the vesicle fusion method is influenced by the degree of hydrophilicity on the substrate surface [2]. Coverage of a lipid bilayer on an moderately annealed $\mathrm{SiO}_{2}$ surface is larger than that on a chemically oxidized one. Since annealing treatment causes irreversible $\mathrm{OH}$ group desorption from the surface [29], the annealed $\mathrm{SiO}_{2}$ surfaces are less hydrophilic than the chemical oxide surfaces. On a strongly hydrophobic surface, a lipid bilayer is not grown but a lipid monolayer [30] or no membrane [3] is formed. These experimental results indicate that an appropriate hydrophilicity on a solid surface exists for a bilayer to be formed by the vesicle fusion method. In an aqueous environment, a bound water layer is formed on a hydrophilic solid surface. Mobility of the bound water is much smaller than that of the bulk water. The bound water layer is thickened as the hydrophilicity of the surface increases. Thus, there is a thick bound water layer on a chemical oxide surface. The thick bound water layer prevents vesicles from adhering on the surface. 

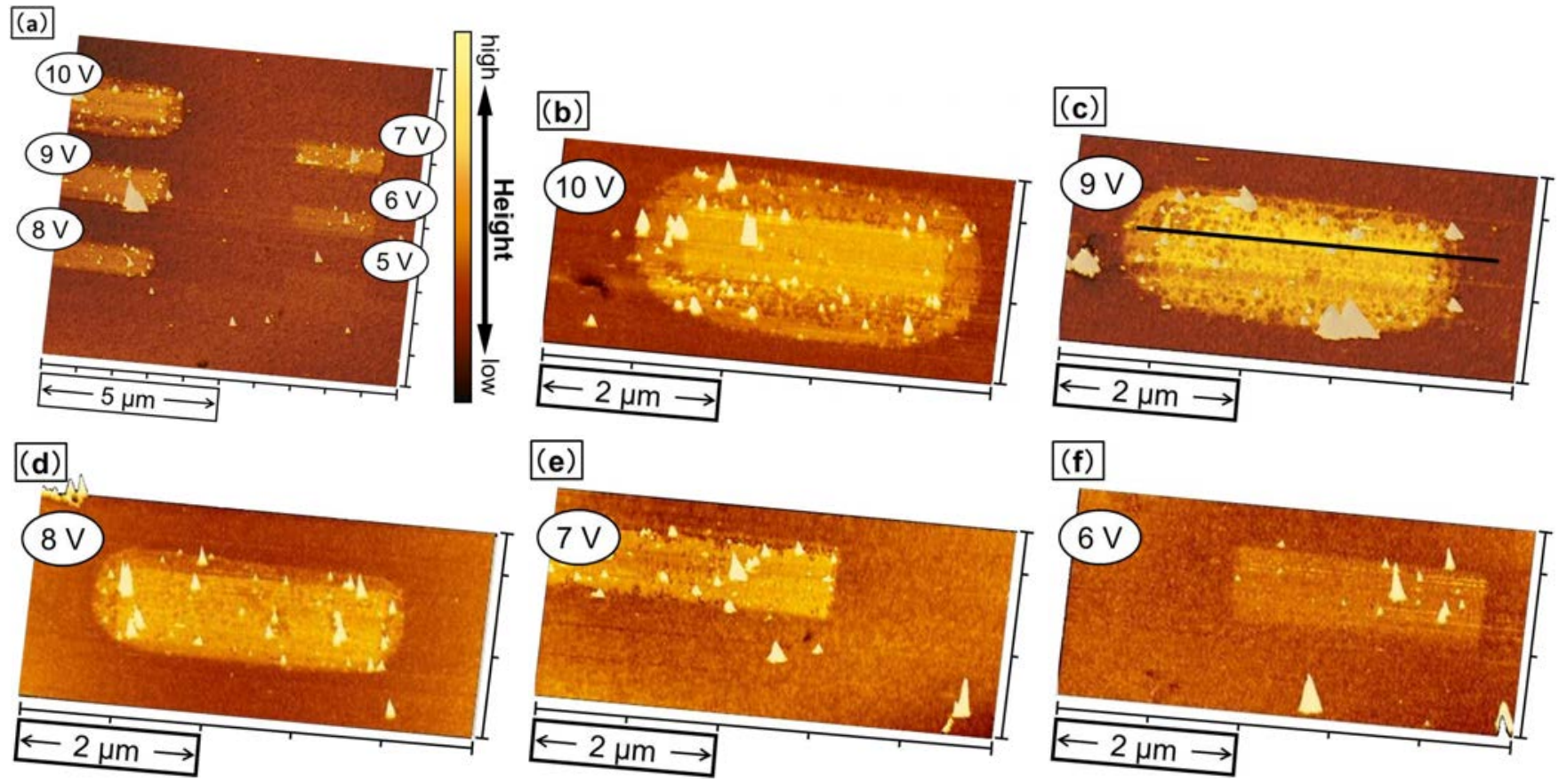

FIG. 4: (a) AFM images of locally anodic-oxidized islands on the OTMS/SiO $/ 2$ Si surface after the DPPC deposition in a buffer solution. The values indicate the applied voltages between the tip and the surface during anodic oxidation. (b)-(f) Magnified images around the oxide islands formed at (b) $10 \mathrm{~V}$, (c) $9 \mathrm{~V}$, (d) $8 \mathrm{~V}$, (e) $7 \mathrm{~V}$, and (f) $6 \mathrm{~V}$, respectively.

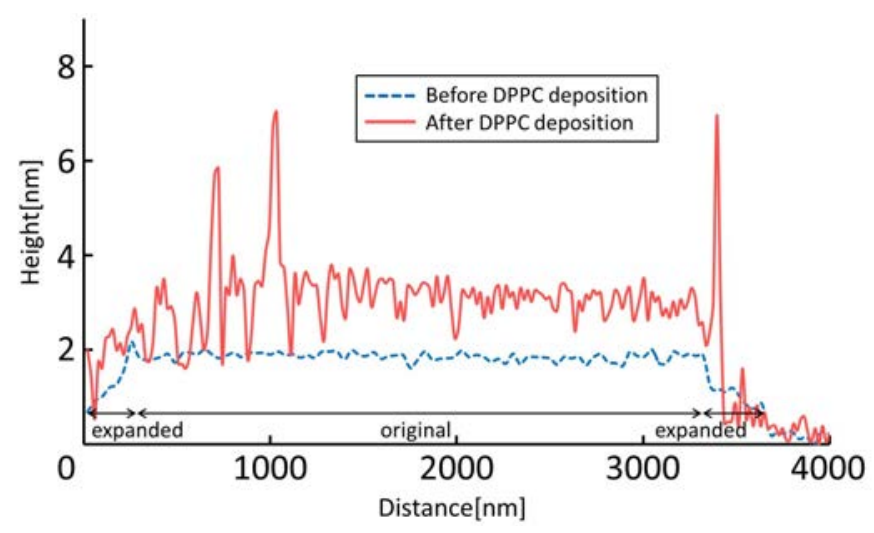

FIG. 5: Cross-sectional view of the oxide island formed at $9 \mathrm{~V}$. Blue broken line indicates cross-section of the oxide island before the DPPC deposition, and red solid line that after the DPPC deposition.

Since the vesicles have to closely approach the surface to rupture and spread on it, lipid bilayer formation is inhibited on strongly hydrophilic surfaces. On the other hand, the bound water layer on a moderately annealed $\mathrm{SiO}_{2}$ surface is thinner than that on the chemical oxide one owing to its weak hydrophilicity. Therefore, the vesicles can adhere and rupture on it. In the present study, hydrophilicity suitable for formation of lipid bilayers are obtained on the original oxide surfaces prepared at 7-9 V and on the all expanded oxide surfaces. The experimental results in this study are summarized in Fig. 7 .

In our results, apparent height of the lipid membrane was somewhat smaller than that of the ideal DPPC bilayer. The DPPC molecule length is about $2.5 \mathrm{~nm}$ in the

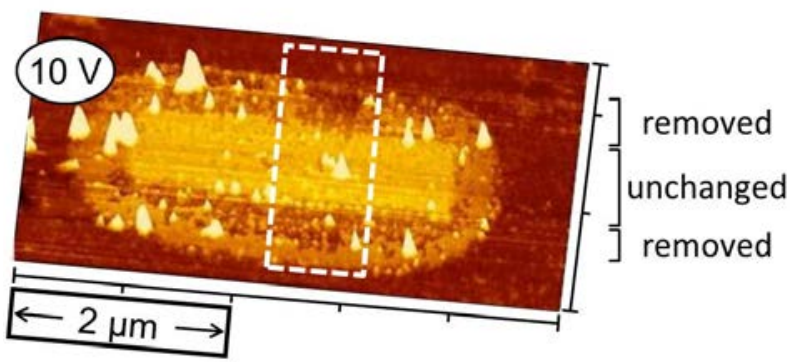

FIG. 6: AFM image after scratching by the tip on the oxide island formed at $10 \mathrm{~V}$.

gel phase, and ideal thickness of a DPPC bilayer is about $5 \mathrm{~nm}$. But, the observed height of the present membrane was about $2 \mathrm{~nm}$. This indicates that the formed membrane is not an ideal bilayer, but either a monolayer or an incomplete bilayer. As shown in Fig. 3, lipid bilayers form on a large area of the oxide surface under the same experimental conditions as that on the anodic oxide islands. Therefore, monolayer formation can be excluded. In our previous report, measured heights of the self-spreading lipid bilayers were influenced by the density of lipid molecule [31]. Though the self-spreading lipid bilayer around the lipid source has an ideal height, the bilayer at the growth front is considerably lower than the ideal one. Since the lipid molecules are supplied only from the source far from the growth front, the density of lipid molecule at the growth front is lower than that around the lipid source. When the density of lipid molecules becomes low, the lipid bilayer structure changes from the closely packed configuration to a weakly bound one. As 


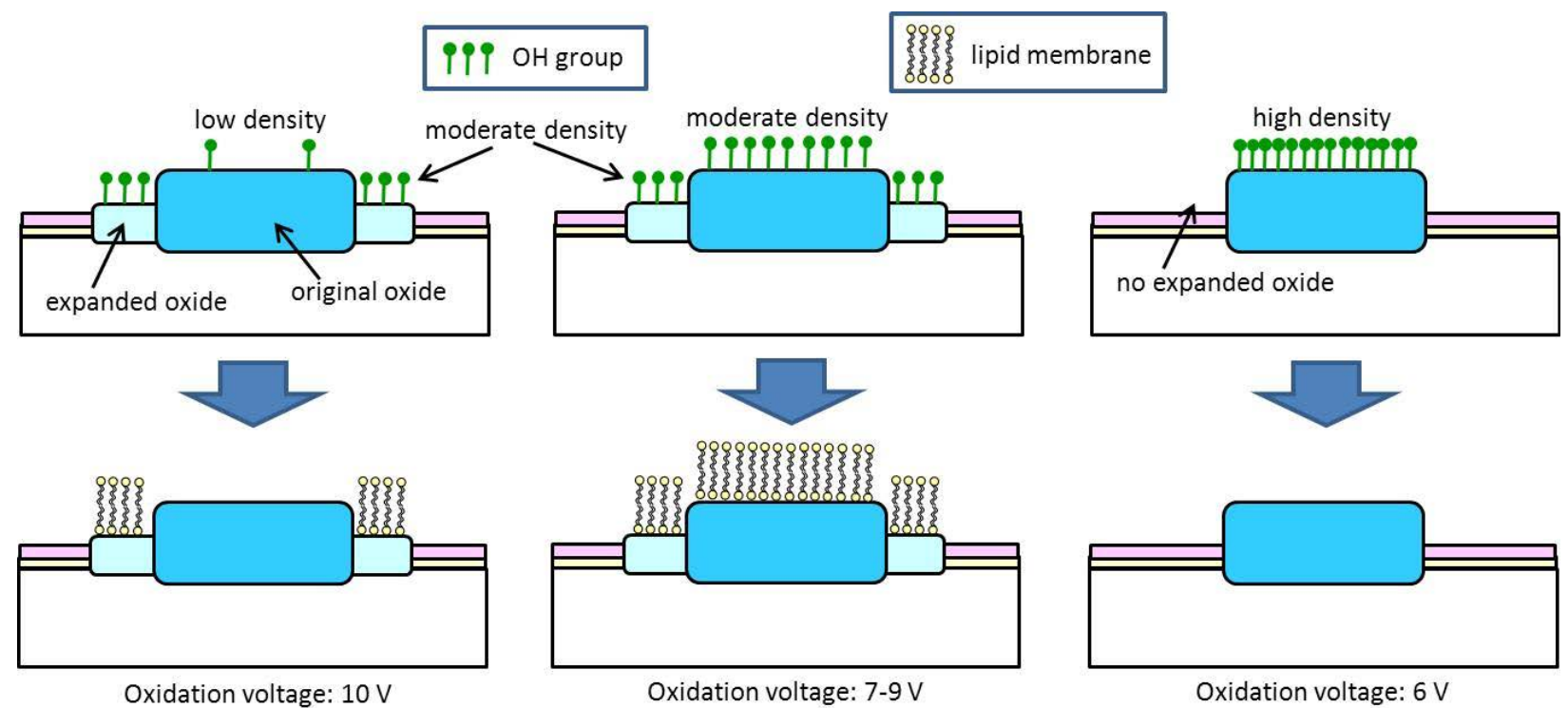

FIG. 7: Schematics of surface chemistry on the oxide islands formed at $10 \mathrm{~V}, 7-9 \mathrm{~V}$, and $6 \mathrm{~V}$, and lipid membrane patterns on those oxide islands.

a result, the hydrophobic attractive interaction between the lipid molecules, which is required to retain the bilayer structure, becomes weak, and the lipid molecules cannot maintain the upstanding bilayer structure, resulting in deformation to the inclined structure. When a lipid bilayer forms on a small oxide island, such as the present experiments, the periphery of the bilayers is unstable because of lack of the adjacent molecules. We can conclude that the observed membrane height is lower than that of the ideal one owing to the deformation of the membrane. Additionally, the formed lipid membrane has many voids because of the low density of the lipid molecule, as shown in Fig. 4.1.

We discuss origin of the difference in membrane formation on the original oxide regions according to Fig. 7. Among the original oxide islands and the base surface, the oxide islands formed at a low voltage $(6 \mathrm{~V})$ exhibited the strongest hydrophilicity, the oxide islands formed at intermediate voltages (from 7 to $9 \mathrm{~V}$ ) the second, oxide islands formed at a high voltage $(10 \mathrm{~V})$ the third, and the OTMS-terminated surface the weakest. Since the OTMS film surface is strongly hydrophobic, no membrane was observed on it. Lipid membranes on the original oxide surface were observed only on the islands formed at the voltages from 7 to $9 \mathrm{~V}$. From the comparison between the present and the previously reported results [32], the island surfaces formed at the voltages from 7 to $9 \mathrm{~V}$ are similar to the moderately annealed $\mathrm{SiO}_{2}$ surfaces or the chemically oxidized surfaces, as far as the hydrophilicity, or the $\mathrm{OH}$ group density, is concerned. When the applied voltage was $6 \mathrm{~V}$, a lipid membrane was not observed. This is probably because hydrophilicity of the oxide islands formed at $6 \mathrm{~V}$ is too strong for the vesicles to approach and rupture [2]. On the other hand, the oxide islands formed at $10 \mathrm{~V}$ is also unsuitable for lipid membrane formation because of its too weak hydrophilicity. The reduction of surface hydrophilicity observed for oxidation at $10 \mathrm{~V}$ is caused by conversion of the $\mathrm{OH}$ groups to the $\mathrm{Si}-\mathrm{O}-\mathrm{Si}$ groups on the oxide surface $[20,21]$. Our result indicates that hydrophilicity of the oxide islands formed at intermediate voltages (from 7 to $9 \mathrm{~V}$ ) is suitable for formation of the lipid membranes.

Next, we focus on chemical properties of the expanded oxide regions according to Fig. 7. Hydrophilicity in these regions was found to be larger than that on the original oxide regions formed at the applied voltage above $7 \mathrm{~V}$, as shown in Fig. 2(b). Oxidant in local anodic oxidation is $\mathrm{OH}^{-}$ions generated in the meniscus, and the expanded region is formed by the $\mathrm{OH}^{-}$ions that are transported from the area just below the tip. The $\mathrm{OH}-$ ions are transported through the meniscus, diffused in the $\mathrm{SiO}_{2}$ layer, and finally reacted with $\mathrm{Si}$ at the $\mathrm{SiO}_{2} / \mathrm{Si}$ interface. When the applied voltage is high, electric field is also large and the $\mathrm{OH}^{-}$ions are diffused further compared with the case of low applied voltages. Therefore, the expanded oxide area is larger when a higher voltage is applied. When the applied voltage is low, conversion of the $\mathrm{OH}$ groups to the $\mathrm{Si}-\mathrm{O}-\mathrm{Si}$ groups does not frequently occur even on the original oxide regions. Thus, hydrophilicity of the expanded oxide region is similar to that of the original oxide formed at lower applied voltages. Behavior of lipid membrane formation on the oxide island formed at $10 \mathrm{~V}$ supports the above interpretation, where the membrane was not observed on the original oxide region whereas it formed on the expanded oxide region, as shown in Fig. 7.

From the above experimental results and discussion, we can conclude that selective adhesion, rupture and fusion of the vesicles occur on the anodic-oxidized surface, though the structure of the formed lipid membranes is not perfect. In local anodic oxidation, ability to drive membrane formation on oxide surfaces depends on the applied voltage. To improve the structure of the lipid bilayers, we should stabilize their peripheries. This can be done by using a raft structure or providing sufficient density of lipid molecules to the anodic-oxidized regions. If these improvements are satisfied, locally anodic-oxidized surfaces with controlled surface chemistry are applicable to patterning of biomolecule adsorption. 


\section{CONCLUSION}

Surface chemistry on a Si substrate was locally controlled by local anodic oxidation using AFM. We prepared several kinds of oxide island that exhibited different hydrophilicity by selecting the applied voltage. We have shown that surface hydrophilicity sensitively affects lipid membrane formation and demonstrated that the lipid membranes are selectively formed on the oxide islands with a moderate hydrophilicity. The formed lipid membranes were incomplete in their structure because of low densities of lipid molecule or presence of the periphery. This disadvantage can be improved by reinforcement of the peripheral structure of the lipid membrane. In local anodic oxidation, arbitrary patterns with different chemical properties can be obtained. We believe that anodic oxidation technique is a useful method to locally modify the surface chemistry toward basic study of biomolecule adsorption on solid surfaces and its application to biodevices.

\section{Acknowledgments}

This work was partly supported by a Grant-in-Aid for Scientific Research from the Ministry of Education, Culture, Sports, Science and Technology. The authors thank Mr. Kashiwase for his helpful support.
[1] R. H. Al-Shakhshir, F. E. Regnier, J. L. White, and S. L. Hem, Vaccine 13, 41 (1995).

[2] R. Tero, H. Watanabe, and T. Urisu, Phys. Chem. Chem. Phys. 8, 3885 (2006).

[3] T. Isono, H. Tanaka, and T. Ogino, e-J. Surf. Sci. Nanotech. 5, 1 (2007).

[4] M. Veiseh, Y. Zhang, K. Hinkley, and M. Zhang, Biomed. Microdevices 3, 45 (2001).

[5] C. D. W. Wilkinson, A. S. G. Curtis, and J. Crossan, J. Vac. Sci. Technol. B 16, 3132 (1998).

[6] D. Falconnet, D. Pasqui, S. Park, R. Eckert, H. Schift, J. Gobrecht, R. Barbucci, and M. Textor, Nano Lett. 4, 1909 (2004).

[7] L. A. Chrisey, C. E. O'Ferrall, B. J. Spargo, C. S. Dulcey, and J. M. Calvert, Nucl. Acids Res. 24, 3040 (1996).

[8] T. Thundat, L. A. Nagahara, P. I. Oden, S. M. Lindsay, M. A. George, and W. S. Glaunsinger, J. Vac. Sci. Technol. A 8, 3537 (1990).

[9] J. A. Dagata, J. Schneir, H. H. Harary, C. J. Evans, M. T. Postek, and J. Bennett, Appl. Phys. Lett. 56, 2001 (1990).

[10] H. C. Day and D. R. Allee, Appl. Phys. Lett. 62, 2691 (1993).

[11] M. Yasutake, Y. Ejiri, and T. Hattori, Jpn. J. Appl. Phys. 32, L1021 (1993).

[12] Y. Mo, Y. Wang, and M. Bai, Physica E 41, 146 (2008).

[13] H. Sugimura, T. Uchida, N. Kitamura, and H. Masuhara, J. Phys. Chem. 98, 4352 (1994).

[14] C. Huh and S.-J. Park, J. Vac. Sci. Technol. B 18, 55 (2000).

[15] E. S. Snow, D. Park, and P. M. Campbell, Appl. Phys. Lett. 69, 269 (1996).

[16] Z. Jiao, H. Zhang, M. Wu, H. Guo, J. Wang, B. Zhao, and G. Ding, J. Mater. Sci.: Mater. Electron. 20, 177 (2009).

[17] F. Marchi, V. Bouchiat, H. Dallaporta, V. Safarov, D.
Tonneau, and P. Doppelt, J. Vac. Sci. Technol. B 16 2952 (1998).

[18] Y. Mo, W. Zhao, D. Huang, F. Zhao, and M. Bai, Ultramicroscopy 109, 247 (2009).

[19] J. Han, D. Kasahara, T. Ichii, K. Murase, and H. Sugimura, J. Vac. Sci. Technol. B 27, 928 (2009).

[20] Y. Kashiwase, T. Oya, and T. Ogino, Jpn. J. Appl. Phys. 47, 6105 (2008).

[21] Y. Kashiwase and T. Ogino, Jpn. J. Appl. Phys. 48, 056512 (2009).

[22] G. Mayer and N. M. Amer, Appl. Phys. Lett. 57, 2089 (1990).

[23] M. Binggeli and C. M. Mate, Appl. Phys. Lett. 65, 415 (1994).

[24] H. Sugimura, N. Nakagiri, and N. Ichinose, Appl. Phys. Lett. 66, 3686 (1995).

[25] M. Fujihira, D. Aoki, Y. Okabe, H. Takano, H. Hokari, J. Frommer, Y. Nagatani, and F. Sakai, Chem. Lett. 7, 499 (1996).

[26] A. Opitz, S. I.-U. Ahmed, J. A. Schaefer, and M. Scherge, Surf. Sci. 504, 199 (2002).

[27] O. Sneh, M. A. Cameron, and S. M. George, Surf. Sci. 364, 61 (1996).

[28] G. Maulucci, M. De Spirito, G. Arcovito, F. Boffi, A. C. Castellano, and G. Briganti, Biophys. J. 88, 3545 (2005).

[29] I.-S. Chuang and G. E. Maciel, J. Phys. Chem. B 101, 3052 (1997).

[30] S. Terrettaz, T. Stora, C. Duschi, and H. Vogel, Langmuir 9, 1361 (1993).

[31] T. Isono, T. Ikeda, and T. Ogino, Langmuir 26, 9607 (2010).

[32] M. Nakamura and T. Ogino, Jpn. J. Appl. Phys. 50, 035202 (2011). 